\title{
Introduction
}

\section{Tethered cord}

\author{
Charles Kuntz IV, M.D., ${ }^{1}$ and Tae Sung Park, M.D. ${ }^{2}$
}

${ }^{1}$ Department of Neurosurgery, University of Cincinnati Neuroscience Institute, University of Cincinnati College of Medicine, Mayfield Clinic and Spine Institute, Cincinnati, Ohio; and 'Department of Neurosurgery, St. Louis Children's Hospital, Washington University, St. Louis, Missouri

For over 100 years physicians have recognized an association between spinal dysraphism and neurological deterioration. In the last 20-40 years, surgeons have begun to elucidate the pathophysiology involving the tethered cord. Despite the relative plasticity of the human nervous system, the spinal cord has a limited ability to accommodate acute and chronic tension. With a better understanding of the pathophysiology, surgeons are beginning to develop novel treatment strategies for the tethered cord.

This issue of Neurosurgical Focus presents several timely reviews of the history, embryology and etiology, and the pathophysiology and treatment of the tethered cord, 11 articles in total. Highlights include a review article with a historical perspective and analysis of neural embryology by Hertzler and colleagues. The Barrow Neurological Institute and University of Michigan neurosurgical groups provide an appraisal of the pathophysiology of pediatric and adult tethered cord syndrome.

Pouratian and colleagues report on electrophysiologically guided untethering of the spinal cord after perinatal repair of a myelomeningocele. The authors review their surgical technique with the identification of an "autonomous placode" in 6 patients. The University of Washing- ton group provides a unique case report on human motor evoked potential changes following spinal cord transection. The spinal cord was transected in 4 quadrants, and motor evoked potentials were lost unilaterally as each anterior quadrant was sectioned.

Mummaneni's group, from the University of California, San Francisco, is pioneering minimally invasive tethered cord release. They provide a comparison of the latter with the mini-open approach. Their critical assessment of the minimally invasive approach is refreshing. Shih and colleagues provide a review of the current management of recurrent adult tethered cord syndrome from detethering to vertebral column shortening. Hsieh and colleagues analyze the clinical outcomes obtained in patients reported on in the literature and who underwent a posterior vertebral column subtraction osteotomy for tethered cord syndrome. The discussion includes a comparison of the outcomes following detethering and vertebral column shortening.

A fascinating report from Samdani and colleagues calls into question the need to perform a spinal cord untethering procedure in cases involving myelomeningocele in which patients are undergoing scoliosis corrective surgery and who do not present with the clinical symptoms of a tethered cord, even though there is radiographic evidence of tethering. The issue also includes a review by Shin and Krishnaney on the rare tethred cord secondary to idiopathic ventral spinal cord herniation and a case report by Gupta, Heary, and Michaels showing reversal of long-standing neurological deficits after the late release of a tethered spinal cord.

We hope that you enjoy this issue of Neurosurgical Focus devoted to the tethered cord. (DOI: 10.3171/ 2010.7.FOCUS.Intro) 